Results: The study included 5062 PsA patients. Data showed an increase in use of bDMARDs in recent years (Fig 1). The trend in increasing use of bDMARDS was not affected by age, sex, and ethnicity. Etanercept was the most commonly used bDMARD (10.1\%) over time, whereas, methotrexate was the most commonly used cDMARD (25.9\%). A decrease in use of infliximab was noted along with the introduction of adimumab, golimumab and ustekinumab. Notably, a decrease in the utilization of cDMARDs, but not corticosteroid use, was seen with the advent of bDMARDs (Fig 2). However, this decrease in cDMARD use was not observed in patients treated with infliximab [Correlation Coefficient $2.69 \%(-47.5 \%, 51.6 \%), P=0.921]$.Conclusion: More PsA patients are using bDMARDs in recent years in lieu of cDMRADS. This change in PsA management highlights a need for further research on the relative safety efficacy and cost of biologic agents to better guide evidence-based treatment decisions.

Disclosure of Interests: Amir Haddad: None declared, Faten Tatour: None declared, Tal Gazitt: None declared, Ilan Feldhamer: None declared, Irina Bergman: None declared, Arnon Cohen Grant/research support from: Prof. Arnon Cohen received research grants from Janssen, Novartis and AbbVie and Sanofi, Consultant for: Prof. Arnon Cohen served as a consultant, advisor for AbbVie; Amgen; Boehringer Ingelheim; Dexcel pharma; Janssen, Lilly; Neopharm; Novartis, Perrigo; Pfizer; Rafa; Sanofi, Speakers bureau: Prof. Arnon Cohen served as speaker for AbbVie; Amgen; Boehringer Ingelheim; Dexcel pharma; Janssen, Lilly; Neopharm; Novartis, Perrigo; Pfizer; Rafa; Sanofi, Devy Zisman Grant/research support from: Dr D Zisman received research grants from Pfizer, Janssen, Consultant for: Dr D Zisman served as a consultant, for Pfizer, Abbvie, Novartis, EllieLilly, Sanofi, Speakers bureau: Dr D Zisman served as a speaker for AbbVie, Janssen, Lilly, Novartis, Pfizer, Sanofi DOI: 10.1136/annrheumdis-2019-eular.6001

\section{AB0788 LUPUS CO-MORBIDITY IN PATIENTS WITH PSORIATIC ARTHRITIS: A POPULATION-BASED CASE- CONTROLLED STUDY}

Danielle Korkus ${ }^{1}$, Tal Gazitt ${ }^{2}$, Ilan Feldhamer ${ }^{3}$, Idit Lavi ${ }^{4}$, Amir Haddad ${ }^{2}$ Erez Batat ${ }^{3}$, Sari Greenberg-Dotan ${ }^{3}$, Arnon Cohen ${ }^{3,5}$, 1,2Devy Zisman ${ }^{*}$. ${ }^{1}$ The Ruth and Bruce Rappaport Faculty of Medicine Technion, Haifa, Israel; ${ }^{2}$ Carmel Medical Center, Rheumatology Unit, Haifa, Israel; ${ }^{3}$ Central Headquarters, Clalit Health Services, Chief Physician's Office, Tel Aviv, Israel; ${ }^{4}$ Carmel Medical Center, Department of Community Medicine and Epidemiology, Haifa, Israel; ${ }^{5}$ Faculty of Health Sciences, Ben-Gurion University of the Negev, Siaal Research Center for Family Medicine and Primary Care, Beer-Sheva, Israel

Background: Patients with psoriasis and psoriatic arthritis (PsA) can develop a variety of comorbidities including metabolic syndrome, diabetes, hypertension, cardiovascular diseases and depression. Comorbidities, in turn, may influence the therapeutic regimen and affect treatment results. Previous studies show a high incidence of coexistence of psoriasis and systemic lupus erythematosus (SLE). Unlike psoriasis, the coexistence of PSA and SLE has been reported only in case reports.

Objectives: To assess the prevalence of SLE in a PsA patient cohort and to compare it to the general population using the database of a large health care provider.

Methods: The study, which was conducted from 2002-2017, is a retrospective study on a PsA cohort consisting of 4,836 PsA patients matched for age and sex with 24,180 randomly selected control patients. Data on this cohort was derived from the database of more than 4.3 million people enrolled in the largest health care provider in Israel, Clalit Health Services. The database was used to extract demographic data, such as age, sex, ethnicity and socioeconomic status; clinical and laboratory manifestations of SLE; medication dispensed and SLE-inducing medications. T-test was used to compare continuous variables and a Chisquare test was used for categorical variables. All tests were 2-sided; $p$ values of $<0.05$ were considered statistically significant.

Results: The PsA study group consisted of 4,836 subjects, at a median age of $56+/-15,2603(53.8 \%)$ of whom were females. The control group consisted of 24,180 subjects matched for age and sex. 18 patients $(0.37 \%)$ in the PsA study group, and 39 patients $(0.16 \%)$ in the control group where diagnosed with SLE $(p=0.002)$. SLE patients without coexisting PsA had higher anti-double stranded DNA (anti-dsDNA) positivity (92.3\% vs $66.7 \%, p=0.022)$ and positive anti-cardiolipin (ACL) antibodies (46.2\% vs $16.7 \%, \quad p=0.041)$. No other significant differences were observed between the two groups in terms of clinical and laboratory manifestations of SLE. PSA patients with concomitant SLE compare to PsA patients without SLE were more often female (100\% vs $53.7 \%$, $\mathrm{p}<0.0001)$, had more osteoporosis $(38.9 \%$ vs $12.8 \%, \mathrm{p}=0.005)$ and were more likely to be treated with beta blockers (27.8\% vs $9.8 \% p=0.027)$. Usage of medications with known potential to induce SLE prior to diagnosis of SLE was higher in the study group of PSA patients (11 out of 18 patients) than in the control group, but there was no difference in SLE manifestations between these two groups. Culprit medications associated with later onset of SLE included proton pump inhibitors (PPI) $[0.27 \%$ in the PsA cohort vs $0.1 \%$ in the control group $(p=0.004)]$, beta blockers $[0.33 \%$ vs $0.16 \% \quad(p=0.011)]$, angiotensin converting enzyme inhibitors (ACE-I) $[0.35 \%$ vs $0.13 \% \quad(p=0.001)]$, thiazide diuretics [0.35\% vs $0.1 \%(p=0.001)]$, anti- tumor necrosis factor (anti-TNF) agents $[0.4 \%$ vs $0.2 \%(p=0.002)]$.

Conclusion: A 2.3 fold increase in the prevalence of SLE in PSA patients than in the control group was found in our study population. There were no significant differences in the clinical and laboratory manifestations of SLE between these two groups. The positive correlates between SLE and PSA may point to common underlying pathogenetic pathways and may affect treatment choices and medication development More research is needed for a better understanding of this diseases association.

Disclosure of Interests: Danielle Korkus: None declared, Tal Gazitt: None declared, Ilan Feldhamer: None declared, Idit Lavi: None declared Amir Haddad: None declared, Erez Batat: None declared, Sari Greenberg-Dotan: None declared, Arnon Cohen Grant/research support from: Prof. Arnon Cohen received research grants from Janssen, Novartis and AbbVie and Sanofi, Consultant for: Prof. Arnon Cohen served as a consultant, advisor for AbbVie; Amgen; Boehringer Ingelheim; Dexcel pharma Janssen, Lilly; Neopharm; Novartis, Perrigo; Pfizer; Rafa; Sanofi, Speakers bureau: Prof. Arnon Cohen served as speaker for AbbVie; Amgen; Boeh ringer Ingelheim; Dexcel pharma; Janssen, Lilly; Neopharm; Novartis, Perrigo; Pfizer; Rafa; Sanofi, Devy Zisman Grant/research support from: D D Zisman received research grants from Pfizer, Janssen, Consultant for: Dr D Zisman served as a consultant, for Pfizer, Abbvie, Novartis, EllieLilly, Sanofi, Speakers bureau: $\mathrm{Dr}$ D Zisman served as a speaker for AbbVie, Janssen, Lilly, Novartis, Pfizer, Sanofi

DOI: 10.1136/annrheumdis-2019-eular.3546

\section{AB0788B RECOMENDATIONS FOR DISEASE ACTIVITY ASSESSMENT AND DEFINITION OF CLINICAL REMISSION IN PSORIATIC ARTHTITIS: A DELPHI- BASED EXPERT CONSENSUS}

Raquel Almodovar ${ }^{1}$, Juan D. Cañete ${ }^{2}$, Eugenio de Miguel $^{3}$, Jose Antonio Pinto Tasende ${ }^{4},{ }^{5}$ Rubén Queiró Silva*. ${ }^{1} 1$ University Hospital Fundación Alcorcón, Madrid., Rheumatology Unit, Madrid, Spain; ${ }^{2}$ Hospital Clínic e IDIBAPS, Barcelona., Barcelona, Spain; ${ }^{3}$ University Hospital La Paz, Madrid, Madrid, Spain; ${ }^{4}$ Complexo Hospitalario Universitario de A Coruña., A Coruña, Spain; ${ }^{5}$ Central University Hospital of Asturias, Asturias, Spain

Background: Patients with psoriasis arthritis (PsA) have heterogeneous clinical presentations, with diverse articular and dermatological features and varied disease courses and outcomes, so the assessment of disease activity implies important difficulties. Although the goal of treatment of PsA is to achieve remission, there is no a universally accepted definition. Objectives: The purpose of this study was to assess agreement by experts on recommended disease activity assessment in PsA patients and to develop a consensus definition of clinical remission.

Methods: A modified Delphi approach was used as a consensus method A scientific committee of experts provided 86 statements that were sub mitted in 2 rounds to a panel of 130 Spanish experts in PsA (in September and October 2018, respectively), addressing issues regarding the current assessment of remission, variables that should be included in the minimal assessment of PsA patients and the definition of remission, and the use of disease activity scores.

Results: The expert panel reached agreement for 53 proposed statements $(61.6 \%)$. There was consensus that the definition of remission in PsA should include: the absence of signs and symptoms, physical well-being, the absence of impact of the disease, the absence of inflammation and progression in imaging tests (radiography and magnetic resonance) and the absence of inflammation measured by biomarkers (ESR, CRP). It is recommended to use some index designed specifically for the assessment of disease activity and the variables that should include the minima assessment of PsA patients are: painful/swollen joints, enthesitis, dactylitis axial involvement, skin and nail involvement, physical function, quality of life, structural damage assessed by imaging techniques, CRP, both patient and physician global assessment, both patient and physician skin assessment and extra-articular manifestations. The use of a treat-to-target (T2T) strategy should be considered in PsA patients, especially in those 